\& Mayer, Leipzig. 3:39-71.

- 1943: Acarina. - In Dr. H. G. Bronn's Klassen und Ordnungen des Tierreichs. Becker \& Erler, Leipzig. 5. Abt. 4. Buch 5. Lief. 1-7:1-1011.

Willmann, C. 1954: Mährische Acari hauptsächlich aus dem Gebiete des mährischen Karstes. - C̈esk. Parasitol. 1:213-232.

Womersley, H. 1935: On some cryptognathid and nicoletiellid Acarina from Australia and New Zealand. - Ann. Mag. Nat. Hist. (10)16:151-154.
Woodroffe, G. E. 1953: An ecological study of the insects and mites in the nests of certain birds in Britain. Bull. Entomol. Res. 44:739-772.

Wrensch, D. L., Kethley, J. B. \& Norton, R. A. 1993: Cytogenetics of Holokinetic Chromosomes and Inverted Meiosis: Keys to the Evolutionary Success of Mites, with Generalizations on Eukaryotes. - In Houck M. A. (ed.): Mites. Ecological and Evolutionary Analyses of Life-History Patterns. Chapman \& Hall, New York and London. pp. 282-343.

\title{
Index to genera and species, Volume 9 (1995)
}

\section{Acarina}

Ixodes ricinus Linnaeus 52

Labidostomma lufeum Kramer (and 27 related taxa including several synomic ones) 211-225

\section{Aranea}

Agyneta rurestris (C. L. Koch) 129, 131

Alopecosa aculeata (Clerek) 129-131

Erigone atra (Blackwell) 29, 131

Hygrolycosa rubrofasciata (Ohlert) 52

Oedothorax apicatus (Blackwell) 129, 131

Silometopus elegans (O. Pickard-Cambridge) 129, 131

Tapinocyba pallens (O. Pickard-Cambridge) 129, 131

Walkenaeria antica (Wider) 129, 131

Xerolycosa nemoralis (Westring) 129-131

Zelotes petrensis (C. L. Koch) 129, 131

\section{Chilopoda}

Geophilus proximus C. L. Koch 213

Lamyctes fulvicornis Meinert 213

\section{Coleoptera}

Several species of Carabidae listed on pp. 76-77 and p. 172, and many of them discussed on pp. 68-72 and pp.

171-174; further 54 species from various families listed on pp. 124-125, 27 species on pp. 163-164 and 195 species on pp. 206-210.

Acanthocinus aedilis (Linnaeus) 112, 113

Africodytes 44

- maximus Biström, sp. n. 43-45

- rubromaculatus Biström 43, 44, 46

- silvestris (Bilardo \& Pederzani) 44

Ahasverus advena (Waltl) 1

Amara equestris (Duftschmidt) 55

Anoplodera livida (Fabricius) 55

Apion laticeps Desbrochers des Loges 55

- spencii Kirby 55
Arhopalus rusticus (Linnaeus) 109, 112-116

Atheta britannice Bernhauer \& Scheerpletz 2

- crassicornis (Fabricius) 2

Bembidion andreae polonicum J. Müller 55

- semipunctatum (Donovan) 55

Bidessus complicatus Sharp 43, 46

Bledius filipes Sharp 62

Carabus menetriesi Hummel 61

Ceutorrhynchus 11

Chrysolina sanguinolenta (Linnaeus) 166

Coccinella septempunctata Linnaeus 128, 131

Coelambus polonicus (Aubé) 55

Colon appendiculatum (Sahlberg) 2

- calcaraium Erichson 2

Cryptophagus lindbergorum Bruce 55

Ctenicera cuprea 164-166

Cucujus cinnaberinus (Scopoli) 62

Dirhagus 39

Dirrhagus lindbergi Palm 39

Dryops griseus (Erichson) 3

- similaris Bollow 3

Dyschirus intermedius Putzeys 62

Elaphrus angusticollis F. Sahlberg 61

Elater pygmaeus Fabricius 40

Eledona agricola (Herbst) 55

Epuraea daica Sjöberg 2

- opalizans J. Sahlberg 2

Gyrophaena orientalis Strand 2

- rugipennis Mulsant \& Rey 2

Harpalus froelichii Sturm 55

Hippodamia notata (Laicharting) 55

Hydaticus aruspex Clark 55

Hydroporus acutangulus Thompson 55

- longicornis J. Sahlberg 55

- neglectus Schaum 55

- septemvittatus Régimbart 4

Hydrosmecta tenella (Mannerheim) 55

Hylobius 128

Hylochares cruentatus (Gyllenhal) 62

Hylurgos palliatus (Gyllenhal) 113 
Ips cumitinus (Eichhoff) 166

- typographus (Linnaeus) 112, 116

Ischnopoda coarctata (Erichson) 55

Isorhipis marmottani (Bonvouloir) 166

Larinus planus (Fabricius) 2

- sturnus (Schaller) 3

Leiodes ferruginea (Fabricius) 3

- picea (Panzer) 3

- rotundata (Erichson) 3

Melanophila acuminata (Degeer) 52

- cyanea (Fabricius) 113, 114, 116

Meligethes 11, 17

- aeneus Fabricius 11-17

Microrhagus 39, 40

- lepidus Rosenhauer 39, 40

- lindbergi (Palm) 39, 40

- pygmaeus (Fabricius) 39, 40

Monochamus sutor (Linnaeus) 112, 113, 116

Monotoma brevicornis Motschulsky 1, 3

- testacea Motschulsky 1, 3

Mycetoporus brucki Pandelle 1

Nebrioporus abyssinicus (Sharp) 4

- tellinii (Régimbart) 4

Nanosella 52

Ophonus puncticollis (Paykull) 55

Oxypteris (Melanophila) acuminata (Degeer) 109, 115, 116

Pachenoporus pilosus (Rossi) 55

Pissodes 113

- pini (Linnaeus) 112

Pityogenes chalcographus (Linnaeus) 109, 112-115

- quadridens (Hartig) 112, 113

Pogonochaerus fasciculatus (Degeer) 113

Polygraphus poligraphus (Linnaeus) 109, 111-116

Proteinus crenulatus Pandelle 3

- macropterus (Gravenhorst) 3

Psylliodes chrysocephala Linnaeus 11

Pterostichus quadrifoveolatus (Letzner) 109, 115, 116

Ptiliolum caledonicum (Sharp) 55

Rhagium inquisitor (Linnaeus) 112, 113

Rhizophagus puncticollis Sahlberg 55

Rhynchaenus alni (Linnaeus) 55

Semanotus undatus (Linnaeus) 113, 114, 116

Sericoda (Agonum) quadripunctata (Degeer) 109, 115, 116

Tetropium 113, 115

- castaneum (Linnaeus) 114

- fuscum (Fabricius) 114

Tomicus 112,114

- minor (Hartig) 111

- piniperda (Linnaeus) 109, 111-115

Tritoma subbasalis (Reitter) 166

Trypodendron lineatum (Olivier) 112, 113

Uvarus 43

- alluaudi (Régimbart) 43

- betsimisarakus (Guignot) 43

- caprai Pederzani \& Sanfilippo 43

- bidachii Pederzani \& Sanfilippo 43

- laurentius Biström sp. n. 43-45

- nubilus (Régimbart) 43, 46
- peringueyi (Rćgimbart) 43

- retiarius (Guignot) 43

- rivulorum (Régimbart) 43

- strcieleni Biström 43, 44

Yola cuspis Bilardo \& Pederzani 43, 46

\section{Diplopoda}

Archiboreiulus pallidus (Brade-Birks) 213

Nemasoma varicorne C. L. Koch 213

Polyxerus lagurus (Linnaeus) 213

Proteroiulus fuscus (Am Stein) 213

\section{Diptera}

Allodia trectata sensu Plassmann 192

- vernalis Polevoi sp. n. 185, 190, 192

Arctophila superbiens (Muiller) 8

Bellardia cognata (Meigen) 2

- viarum (Robineau-Desvoidy) 1

Boletina minuta Polevoi sp. n. 185-188

- onegensis Polevoi sp. n. 185, 189, 192

- populina Polevoi sp. n. 185, 187, 189, 192

- sciarina Staeger 192

- triangularis Polevoi sp. n. 185, 186, 188

Brachicoma devia (Fallén) 1

Brachyopa bicolor (Fallén) 8

- conica auctt. 8

- panzeri Goffe 8

- pilosa Collin 8

- testacea (Fallén) 8

Brevicornu fasciculatum (Lackschewitz) 192

- fennicum (Landrock) 193

- improvisum A. Zaitzev sp. n. 185, 193, 194

- neofasciculatum A. Zaitzev sp. n. 185, 190, 192

- parafennicum A. Zaitzev sp. n. 185, 191, 193

- proximum-group 193

- setigerum A. Zaitzev sp. n. 185, 190, 193

Calliphora loewi Enderlein 1

- stelviana (Brauer \& Bergenstamm) 2

- vicina Robineau-Desvoidy 2

Chamaesyrphus caledonicus Collin 7

Cheilosia rufimana (Becker) 7

Chironomus 20, 25, 26, 28

- aberratus Keyl 20

- acutiventris acutiventris Wülker, Ryser \& Scholl 19, 20, 25-28

- acutiventris bavaricus Wülker, Ryser \& Scholl 25, 27

- annularius auct. 26, 27

- cingulatus Meigen 25, 26

- clarus Hirvenoja 19-28

- obtusidens Goethebuer 19-21, 25-28

- piger Strenzke 20, 28

- plumosus (Linnaeus) 20

- riparius Meigen 21, 26, 28

- thummi complex 19, 20

- venustus Staeger 25

Chrysogaster maquarti auctt. 8

Chrysops parallelogrammus Zeller 55 
Dasyneura brassicae (Winnertz) 11

Dynatosoma dichaeta Polevoi sp. n. 185, 191, 194

- nigromaculatum Lundström 194

- ussuriense A. Zaitzev 194

Einfeldia 26

Empis punctata (Meigen) 62

Epistrophe 8

- euchroma (Kowarz) 8

- flava Doczkal \& Schmid 7

- melanostoma (Zetterstedt) 8

- melanostomoides auctt. 7

- melanostomoides (Strobl) 8

- ochrostoma auctt. 7

- similis Doczkal \& Schmid 7

Lipoptena cervi (Linnaeus) 164, 166

Mallota cimbiciformis (Fallén) 8

Melanogaster 8

- aerosa (Loew) 8

Melinda coerulea (Meigen) 2

- gentilis Robineau-Desvoidy 6

- viridicyanea (Robineau-Desvoidy) 2

Metasyrphus abiskoensis Dusek \& Laska 5

Microdon devius (Linnaeus) 8

- indistincta Polevoi sp. n. 185-187

Oebalia minuta (Fallén) 2

Oenesia floralis Robineau-Desvoidy 6

Paragus albifrons Fallén 7,8

- majoranea Rondani 7,8

Phronia cornuta Lundström 194

-myrilli Polevoi sp. n. 185, 193, 194

Pipizella varipes Meigen 5

- viduata (Linnaeus) 5

Platycheirus complicatus Becker 5

- hyperboreus Staeger 5

- latimanus (Wablberg) 5

Protocalliphora azurea (Fallén) 2

Pterocheilus phaleratus (Panzer) 62

Rhamphomyia marginata (Fabricius) 164

Sphaerophoria 7

- abbreviata Zetterstedt 6-8

- bankowkae Goeldlin 5, 8

- batava Goeldlin 7

- boreoalpina Goeldlin 7

- fatarum Goeldlin 5, 7, 8

- laurae Goeldlin 7

- virgata Goeldlin 6,8

Thyrsocnema incisilobata (Pandelle) 2

Urophora cardui (Linnaeus) 164, 166

\section{Ephemeroptera}

Baetis rhodani (Pictet) 91, 92, 94-97

- subalpinus Bengtsson 91, 92, 97

Caenis horaria (Linnaeus) 96

Centroptilum luteolum (Müller) 91, 97

Ephemerella murconata (Bengtsson) 91-93, 97

Heptagenia sulphurea (Müller) 91, 92, 97

Nixe joernensis (Bengtsson) 92, 96, 97
Procloeon bifidum (Bengisson) 92, 96, 97

\section{Heteroptera}

102 taxa of Lygaeidae from Iraq (with at Ieast some of their synonymic names) are listed on pp. 29-38.

Acanthosoma haemorrhoidale (Linnaeus) 164

Acetropis gimmerthalii (Flor) 55

Aradus truncatus Fieber 62

Coptosma scutellatum (Geoffroy) 55

Coranus aethiops Jakovlev 55

Ligyrocoris sylvestris (Linnaeus) 130, 131

Myrmedobia exilis (Fallén) 130, 131

Palomena prasina (Linnaeus) 164

Temnostethus pusillus (Linnaeus) 124

Tritomegas bicolor (Linnaeus) 124

Troilus ruridus (Fabricius) 164

\section{Homoptera}

Calaphis arctica Hille Ris Lambers 120

Eupteryx aurata (Linnaeus) 62

Hephathus nanus (Herrich-Schaeffer) 61

Impatientinum asiaticum Nevsky 120

Impatiens parviflora (Nevsky) 120

Mongolajassus bicuspidatus (J. Sahlberg) 61

Myzus persicae (Sulzer) 121

Neoliturus fenestratus (Herrich-Schaeffer) 61

Psammotettix frigidus (Boheman) 62

\section{Hymenoptera}

27 species of ants discussed on pp. 153-161.

Acantholyda 180

Amauronematus 179

Anergatus atratulus (Schenck) 153

Apis mellifera Linnaeus 140

Bombus 139, 140

- batagiatus Nylander 61

- consobrinus Dablbom 62

- hortorum (Linnaeus) 141

- hypnorum (Linnaeus) 141-146, 148

- lapidarius Linnaeus 139, 141-146, 148

- lucorum (Linnaeus) 141-146, 148

- pascuorum (Scopoli) 141-146, 148

- pratorum (Linnaeus) 141-146

- schrencki Morawitz 166

- semenoviellus Skorikov 61

- subterraneus (Linnacus) 141, 142

- sylvarum (Linnaeus) 164

Camponotus herculeanus Linnaeus 129, 131, 136

- ligniperda (Latreille) 215

Dineura virididorsata Retzius 179

Formica 136

- aquilonia Yarrow 129, 131, 133, 135-137

- fusca Linnaeus 133, 136, 214, 215

- rufa Linnaeus 153 
- rufibarbis Fabricius 133, 136, 137

- sanguinea Latreille 129, 131, 136

Formicoxenus nitidulus (Nylander) 153

Halictus quadricinctatus (Fabricius) 62

Harpagoxenus sublaevis (Nylander) 153

Konowia betulae (Enslin) 2

Lasius 214, 215

- flavus Fabricius 136, 154, 214

- niger Linnaeus 129, 131, 136, 214, 215

- platythorax Seifert 130, 131

Leptothorax acervorum (Fabricius) 129, 131, 153

- interruptus (Schenck) 153

- kutteri Buschinger 153

Myrmica 155, 215

- gallienii Bondroit 154

- hellenica Forel 153, 154

- hirsuta Elmes 153, 155

- laevinodis Nylander 136

- lobicornis Nylander 129, 131, 155

- lonae Finzi 153, 155

- microrubra Seifert 153, 155, 157

- rolandi Bondroit 154

- rubra Linnaeus 154, 155

- ruginodis Nylander 129, 131, 136, 154, 155

- rugulosa Nylander 154

- sabuleti Meinert 153, 155

- scabrinodes Nylander 154

- specioides Bondroit 153

- sulcinodis Nylander 129, 131, 154

Nematus 179

Neodiprion 180

- sertifer (Geoffroy) 178

Pemphredon mortifer Valkeila 62

Polistes nimpha (Christ) 61

Psithyrus 141

Symbiomyma karavajevi Atnoldi 153

\section{Isopoda}

Trichoniscus pusillus (Brandt) 213

\section{Lepidoptera}

19 additional species are listed on page 124 , and 14 on page 164 .

Araschnia levana (Linnaeus) 62

Archiearis 180

Autographa excelsa (Kretschmar) 55, 61
Charadrina grisea (Eversmann) 61

Clossiana thore (Hübner) 62

Colostygia turbata (Hübner) 62

Deltote bankiana (Fabricius) 62

Depressaria pimpinellae Zeller 55

Erastia penthima Erschoff 197

Erebia eyrunale eyryaloides Tengström 61

Estimata herrichschaefferi (Alpheracy) 197

Hyptioxesta 197

- kurenzovi Kononenko 197, 198, 200

- magadanica (Kononenko) 197-200

- penthima (Erschoff) 197, 198, 200

Ipimorpha contusa (Freyer) 55

Lycia 179

Maculinea arion (Linnaeus) 155

Maniola jurtina (Linnaeus) 166

Ochsenheimeria 53

- urella Fischer von Röslerstamm 55

Orthosia 52

Polygonia c-album (Linnaeus) 166

Semiothisa artesiaria (Denis \& Schiffermüller) 55

Victrix umovii (Eversmann) 55

\section{Neuroptera}

Myrmeleon bore Tjeder 135

- formicarius Linnaeus 135

\section{Odonata}

Libellula depressa Linnaeus 164, 166

\section{Orthoptera}

Bryodema tuberculata (Fabricius) 62

Chorthippus pullus (Philippi) 61

\section{Plecoptera}

19 species listed on page 103, and discussed on pp. 99-107.

\section{Psocoptera}

Blaste conspurgata (Rambur) 1

- quadrimaculata (Latreille) 1

\section{Trichoptera}

23 species listed on page 97, and many of them discussed on pp. 91-96. 